\title{
Pathosphysiology of Chronic Myeloid Leukemia, Prognistic Factors and Emerging Treatment Options in a Low Resource Economy
}

\author{
Patrick Olanrewaju Osho ${ }^{1,}$,, Okunnuga Ndidi ${ }^{2}$, Ojo Matilda ${ }^{1}$, Odunlade Olufunke ${ }^{3}$ \\ ${ }^{1}$ Department of Hematology and Immunology, University of Medical Sciences, Akure, Nigeria \\ ${ }^{2}$ Departments of Surgery (Oncology), University of Medical Sciences, Akure, Nigeria \\ ${ }^{3}$ Department of Paediatrics, University of Medical Sciences, Akure, Nigeria
}

Email address:

droshopo@gmail.com (P. O. Osho),droshopo@unimed.edu.ng (P. O. Osho)

${ }^{*}$ Corresponding author

\section{To cite this article:}

Patrick Olanrewaju Osho, Okunnuga Ndidi, Ojo Matilda, Odunlade Olufunke. Pathosphysiology of Chronic Myeloid Leukemia, Prognistic Factors and Emerging Treatment Options in a Low Resource Economy. Cancer Research Journal. Vol. 9, No. 2, 2021, pp. 111-121. doi: $10.11648 /$ j.crj.20210902.15

Received: April 21, 2021; Accepted: May 17, 2021; Published: June 25, 2021

\begin{abstract}
Chronic myeloid leukemia (CML) is a triphasic clonal myeloproliferative disorder characterized by the presence of Philadelphi a chromosome $(\mathrm{Ph})$ in over $95 \%$ of cases alongside excessive accumulation of clonal myeloid cells in hematopoietic tissues. This occurs as a result of reciprocal translocation between the long arms of chromosome 9 and $22 \mathrm{t}$ $(9 ; 22)$ (q34; q11) creating the fusion oncogene BCR-ABL1 which exhibits constitutive tyrosine kinase activity. It is one of the commonest haematological malignancies in low economies around the world including Nigeria. The clinical features of CML are often described in 3 phases namely the chronic phase (CP), accelerated phase (AP), and blastic phase (BP) while CP is the most common stage with progression to AP and BP occurring later. Despite that, prognosis of CML is dependent on phase of disease, age, and response to therapy, the only curative approach in use currently is hematopoietic stem cell transplantation with other drugs being used for cytogenetic responses. This study focuses on the aetiopathophysiology, cytogenetics, molecular biology, clinical/laboratory features and treatment options of CML. Rigorous review of literature on the study was retrieved from relevant oncology journals and textbooks abstracted and indexed in PubMed, Google Scholar, ProQuest, CINAHL, and Science Direct. The study discovered that, resistance of CML to imatinib has been reported with research having reached the advanced stage on the use of alternative drugs (e.g., Nilotinib and Desatinib). There are also potentials for these new drugs to become the treatment choice and first line drugs for the treatment of CML.
\end{abstract}

Keywords: Chronic Myeloid Leukemia, Oncogene, Imatinib, Tyrosine Kinase, Scoring System

\section{Introduction}

Chronic Myeloid Leukemia (CML) is a form of leukemia characterized by an increased and unregulated growth of predominantly myeloid cells in the bone marrow and the accumulation of these cells in the blood. [1]. This usually occurs as a result of reciprocal translocation between the long arms of chromosome 9 and $22 \mathrm{t}(9 ; 22)$ (q34; q11) creating the fusion oncogene BCR-ABL1. CML has 3 phases namely the chronic phase, aggressive phase, and terminal blastic phase [2].

CML has an estimated global incidence of one to two new cases per 100,000 population per year with a male to female ratio of 1.8:1 with a slight male predominance [3]. Incidence of CML rises gradually with age to reach a median of around 60 years. A global estimate of $15 \%$ of all new cases of leukemia are chronic myeloid leukemia [4]. The median age of CML patients in low-income countries including Nigeria and other African countries is 38 years compared to 67 years in developed countries [6] with a prevalence rate of $30 \%$, Asian (36\%). In Ondo State, south western Nigeria, the prevalence is $15.6 \%$ in adults and $10 \%$ in children. CML may affect any age group; the peak incidence is between 40 and 60 years with the median age at diagnosis being 53 years in the Western world [5]. CML in children still occurs rarely 
constituting $2 \%$ of all leukaemias seen in childhood, however there has been an increased incidence over the last year.

The first case of CML was discovered by Edinburgh pathologist John Hughes Bennet and Rudolf Virchow in 1845. In 1827, Ernst Neumann observed that leukemia cells originate from bone marrow. A real quantum leap was however the discovery of small G-group chromosome now called Philadelphia chromosome by Nowell and Hungerford in 1960 and by 1973, Rowley showed this to be a translocation between long arms of chromosome 9 and 22 [7].

The treatment of CML has greatly evolved over the years. Therapy developed slowly with the use of arsenic in 1865 which was followed by splenic irradiation in 1920. Effective control of blood counts became feasible in 1959 and was followed by use of hydroxyl-urea in 1969. In early 1970s, interferon- $\alpha$ was found to induce durable complete cytogenetic responses. However, the approval of imatinib mesylate in 2002 by the US Food Drug Agency (FDA) has changed the natural history of CML changed dramatically [8]. For the patients who are resistant to imatinib, second line inhibitors are an effective strategy.

CML has been conventionally managed in Nigeria with cyclophosphamide, busulfan, and hydroxyurea. The Max Foundation was highly instrumental to the setting up of the Novartis Pharmaceuticals-supported Glivec International Patient Assistance Program (GIPAP), which provides free access to imatinib for many patients from several developing countries, including Nigeria [9]. This article therefore focuses on the aetiopathophysiology, clinical laboratory features, and treatment of CML.

\section{Methodology}

The current study is a reviewed study in which rigorous literature search was conducted on the topic. A review of literature on the study was retrieved from related oncology journals and textbooks abstracted and indexed in Google Scholar, ProQuest, PubMed, CINAHL, and Science Direct as well as reputable online articles related to the study. Total of 70 (seventy) publications were consulted out of which 55 (fifty-five) were eventually used in the study. Out of this, 45 (forty-five) were sourced from online journals while, 10 (ten) were from online books.

\subsection{Aetiopathophysiology of Chronic Myeloid Leukemia}

Specific genetic or environmental factors can result in the fusion of breakpoints of chromosome 9 in the ABL gene with certain breakpoints on chromosome 22 in the BCR gene. No concrete evidence has been provided for the genetic origin of CML, [10] however there is a higher risk of developing CML due to chemical exposure to benzene, organic solvents, alkylating agents and other chemotherapeutic agents and high doses of irradiation. Infectious agents or hereditary factors has not been implicated in CML development. [11]

During disease progression, $\mathrm{Ph}$ positive cells may acquire additional cytogenetic defects with underlying mechanisms not fully understood. Some studies have shown tumor suppression genes and leukemic oncogenes to be responsible for this. [12, 14, 15]

CML is characterized by presence of Philadelphia chromosome in different cell lines resulting from reciprocal translocation between chromosomes 9 and 22. This chromosome can also be detected in other patients with acute lymphoblastic anaemia (ALL) and in some cases acute myeloid leukemia (AML). Patients with CML usually exhibit a BCR/ABL protein of molecular mass $210 \mathrm{kDa}$ which differs from $190 \mathrm{kDa}$ found in ALL patients and $230 \mathrm{kDa}$ found in Chronic neutrophilic leukemia (CNL) patients. Increased tyrosine kinase activity has been connected with this disease due to the translocation of $\mathrm{ABL}$ and BCR chromosomes. The $\mathrm{BCR} / \mathrm{ABL}$ fusion gene plays a major role in the pathogenesis of CML. This gene encodes a protein of molecular weight 210 $\mathrm{kDa}$ with greater tyrosine kinase activity which affects cell growth, differentiation, decreased apoptosis, and poor adherence of leukemic cells to the bone marrow. Recent data has suggested that $\mathrm{BCR} / \mathrm{ABL}$, an oncogene also contributes to an upregulated expression of angiogenic growth factors [12].

The translocation is passed by the leukemic stem cell population, such that both myeloid and lymphoid progeny may carry $t(9 ; 22)$ in an affected individual. In a smaller number of cases, the Philadelphia chromosome cannot be seen under microscopic karyotipic analysis but is visible through Polymerase Chain Reaction (PCR) or Fluorescence in-situ Hybridization (FISH). These patients usually have some haematological features typical of myelodysplasia. $\mathrm{Ph}$ negative CML behaves clinically like the Ph-positive CML but varies at a molecular level with different treatments [13].

\subsection{Cytogenetics and Molecular Biology}

CML diagnosis depends majorly on detection of $\mathrm{Ph}$ chromosome with either cytogenetic analysis or PCR. The position of the genomic breakpoint region in $\mathrm{ABL}$ gene is variable and most times always located in the intron between exons 1 and 2 as opposed to exons 13-15 in breakpoint region of BCR gene. The resulting fusion transcripts are named e13a2 and e14a2 transcripts, both of which may coexist in one individual due to alternate splicing. These transcripts encode for a $210 \mathrm{kDa}$ protein product (p210). A breakpoint immediately downstream of the first $\mathrm{BCR}$ exon produces the e1a2 transcript, which encodes the p190 BCR-ABL1 protein. This is more commonly seen in Ph-positive ALL and rare in CML. Rarely, the BCR breakpoint occurs between exons 1720 , leading to the larger $\mathrm{p} 230$ protein from the e19a2 transcript, associated with a more prominent neutrophilia and thrombocytosis. Other fusion transcripts such as e6a2, e8a2, and e18a 2 have also been seen which encode for the following rare variant proteins p195, p200, and p225. [15, 16]

$\mathrm{BCR} / \mathrm{ABL}$ activates 3 major pathways through various signaling molecules namely the p21Ras, the PI-3-kinase-Akt and STAT5-pathway. These pathways exhibit several cross reactions with numerous substrates and molecules. BCR exists in a complex with SOS being a nucleotide exchange factor for Ras which in turn leads to phosphorylation and activation of MAP-kinases. In PI3-kinase-Akt-mTOR 
pathway is involved in $\mathrm{BCR} / \mathrm{ABL}$-dependent expression of several critical effector molecules including angiogenic cytokine. Also, BCR/ABL phosphorylates STAT5 and that activation of STAT5 contributes to growth and viability of BCR/ABL-transformed cells. [17, 18].

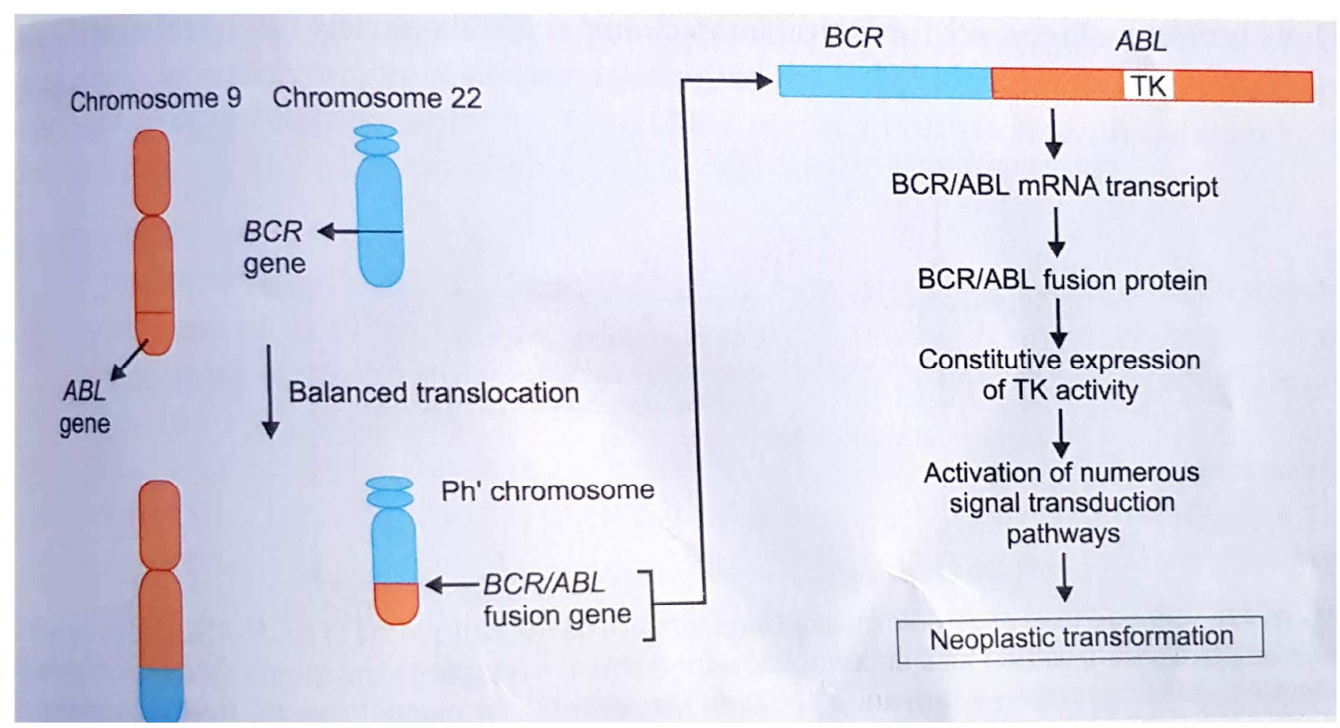

Figure 1. Formation of Philadelphia chromosome and BCR/ABL fusion gene product. Mechanism of leukaemogenesis is shown on right side. (Essentials of Haematology, Shirish M. Kawthalkar).

\subsection{Clinical Features of CML}

The clinical features of CML are often described in 3 phases. The two commonly used classifications are the WHO classification and European Leukemia Net (ELN) classification. An important differentiation between these 2 is the higher myeloblast count $(30 \%)$ as opposed to $20 \%$ in $\mathrm{WHO}$ classification [19]. Most countries particularly in Nigeria however use the WHO classification. WHO criteria for the diagnosis of various phases of CML is shown in Table 1 below:

\subsection{Phases of CML}

There are 3 phases of CML namely the chronic, accelerated, and blast crisis.

Chronic phase: this stage is the most common stage among newly diagnosed CML patients. Diagnosis during this stage is often accidental with patients often coming in for an unrelated medical problem. Patients may present with weight loss, night sweats, fatigue, and splenomegaly. Haematological abnormalities include leukocytosis ranging from 20-200 x 10 $/ 1$, PCV between 25-30\%. Platelet number are usually increased in the range of $300-600 \times 10^{9} / 1$. Anaemia is present in virtually all patients at diagnosis and usually ranges between mild to moderate in degree [20]. Number of leucocyte count is usually directly proportional to size of spleen, basophil count, and percentage of blast cells. Nucleated red cells are occasionally present in the circulation of some patients with alkaline phosphatase in neutrophils being markedly reduced. Bone marrow examination is not compulsory to confirm CML diagnosis but is however carried out to rule out accelerated or blastic phase. Myeloid: erythroid ratio is 10:1 to 50:1 with the normal ratio being 2:1 to 4:1. Myeloblast count is usually below $10 \%$ with bone marrow aspiration revealing hypercellular marrow with markedly increased granulopoiesis [9].
Accelerated phase: this phase is characterized by increased resistant of blood cell counts and organomegaly to chemotherapy and impaired differentiation. According to WHO, there may be increased percentage of blast cells (10$19 \%)$ as well as increasing leukocytosis, basophilia (>20\%), and splenomegaly. Persistence of thrombocytopenia $(<100 \mathrm{x}$ $\left.10^{9} / 1\right)$ unrelated to therapy also occurs alongside cytogenetic evidence of clonal evolution such as duplication of $\mathrm{Ph}$ chromosome, trisomy 8 and 19 [21].

Blast crisis: this phase is characterized by a rapid increase in blast counts alongside marrow failure as a result due to uninhibited growth of myeloid precursors with absence of maturation or differentiation. The blastic phase of CML is majorly indistinguishable from other cases of acute leukemic transformation. According to WHO classification, it is diagnosed by any of the following: Blast in peripheral blood or bone marrow $>20 \%$, blast proliferation at a site other than bone marrow, focal clustering of blasts in bone marrow. Blast crisis in CML may either be myeloid $(70 \%)$ or lymphoid (30\%) resulting in AML (Acute Myeloid Leukemia) or ALL (Acute Lymphoblastic Leukemia) [22].

However, cases of misdiagnosis of chronic stage of CML is common in developing countries like Nigeria where there is a shortage of expertise in Haematology. Most primary health care workers tend to confuse the clinical symptoms of this stage of CML with sepsis (Lukaemoid reaction). Lukaemoid reaction can occur in infections, inflammation, and malignancy showing leukocytosis from left shift usually metamyelocyte and myelocte stage. Occasionally, blasts may occur but usually less than $2 \%$ with the major features of leukaemoid reaction being absence of splenomegaly, or basophilia, presence of toxic granules in neutrophils normal or increased NAP score, and absence of Ph' chromosome [23]. Differential diagnosis of CML and leukaemic reaction 
is shown in the table 2 below:

Some biochemical changes that can be seen in diagnosis of CML patients include: increase in serum alkaline phosphatase, LDH, Potassium level, vitamin B12 and B12 binding capacity. Serum uric acid may also be raised alongside hypercalcemia due to bone destruction [24].

Table 1. WHO criteria for the diagnosis of various phases of CML.

\begin{tabular}{lll}
\hline CML chronic phase & CML accelerated phase & CML blast phase \\
\hline & Increasing spleen size and WBC unresponsive to therapy. & \\
& Cytogenetic evidence of clonal evolution. & Blasts $\geq 20 \%$ in pb or bm. Extramedullary \\
Granulocytosis in the presence of Ph- & Blasts $10-19 \%$ in Pb and/or bm & blast proliferation. \\
chromosome and/or BCR/ABL. & Peripheral blood basophils $\geq 20 \%$ & Large foci or clusters of blasts in the bone \\
No signs of CML-AP or $-\mathrm{BP}$ & Persistent thrombocytopenia $\left(<100 \times 10^{9} / \mathrm{L}\right)$ & marrow biopsy \\
& unrelated to therapy or & \\
& Persistent thrombocytosis $\left(>1000 \times 10^{9} / \mathrm{L}\right)$ & \\
& unresponsive to therapy & \\
\hline
\end{tabular}

Sillaber et al., 2003

Table 2. Differences between chronic myeloid leukemia and leukaemoid reaction.

\begin{tabular}{lll}
\hline Parameter & Chronic myeloid leukemia & Leukaemoid reaction \\
\hline $\begin{array}{l}\text { Clinical features } \\
\text { Peripheral blood }\end{array}$ & Splenomegaly & As per underlying disease \\
a. Leucocyte count & Usually $>100,000 / \mathrm{cmm}$ & Usually $<50,000 / \mathrm{cmm}$ \\
b. Myelocyte and neutrophil peak & Present & Absent \\
c. Basophilia, eosinophilia, monocytosis & Present & Absent \\
d. 'Toxic' granules & Absent & Present \\
NAP score & Low & Normal or Increased \\
Bone marrow examination & Trilineage hyperplasia & Myeloid hyperplasia \\
Genetic analysis & Ph' chromosome or BCR/ABL gene rearrangement & Normal \\
\hline
\end{tabular}

Shirish M. Essentials of Haematology, 2013

\subsection{Other variants of Chronic Myeloid Leukemia}

A couple of other variants of CML include:

Ph-negative chronic myeloid leukemia: this occurs in about $6 \%$ of CML patients. These patients could either have the $\mathrm{BCR} / \mathrm{ABL}$ gene on chromosome $9 \mathrm{q}$ or no $\mathrm{BCR} / \mathrm{ABL}$ gene at all. They may lack basophilia, blasts, and myelocyte peaks in leucocyte differential. These patients respond poorly to Imatinib, IFN-a, or hydroxyurea and overall their survival is inferior to that of $\mathrm{Ph}$-positive patients [25].

Chronic myelomonocytic leukemia: this is a rare myeloproliferative condition usually predominant in elderly men. It is characterized by enlarged spleen, lack of $\mathrm{Ph}$ ' chromosome, prominence of blood monocytosis with high monocyte numbers $\left(50 \times 10^{9} / \mathrm{L}\right)$. Thrombocytopenia is common with basophilia and eosinophilia being absent. It is usually treated using hypomethylating agents such as 5azacitidine and decitabine [26].

Chronic neutrophilic leukemia: this is an extremely rare incidentally diagnosed disorder. Patients usually present with raised blood neutrophil count without immature granulocytes, basophilia, or eosinophilia. The marrow is hypercellular but cytogenic studies are usually normal. Treatment is usually done using hydroxyurea and oral chemotherapy agents [27]

Eosinophilic leukemia: this is a rare condition characterized by pure proliferation of mature cells. Most patients with idiopathic hypereosinophilia have an interstitial deletion of chromosome 4 that results in a FIP1L1-PDGFRA fusion gene. These patients are often responsive to low doses of imatinib. In cases with no fusion gene, the number of immature cells in blood and marrow is increased and finding of clonal cytogenic abnormality is a strong indication of eosinophilic leukemia [28].

Juvenile chronic myeloid leukemia: this is a rare condition affecting children under the ages of 12 years with clinical features such as skin rashes, lymphadenopathy, hepatosplenomegaly and recurrent infections. Leucocyte numbers are increased with variable number of blast cells in the peripheral blood. Such cells are hypersensitive to GMCSF in vitro. The blood film shows monocytosis with elevated levels of haemoglobin F. Neutrophil alkaline phosphatase score is normal and no $\mathrm{Ph}$ ' chromosome is detected. The marrow is hypercellular but usually lacks chromosomal abnormalities. The disease responds poorly to standard cytotoxic drugs but patient may benefit from allogenic SCT [29, 30].

\subsection{Prognostic Factors, Survival, and Scoring Systems}

The prognosis of CML patients has continuously changed over the years with development of new drugs. Survival rate or outcome of CML depends solely on the phase of the disease at diagnosis which also varies from person to person, with reduction in survival rates as the disease further advances if left untreated. Prognosis of CML usually occurs in the chronic phase of the disease running a stable course with a median survival time of patients ranging between 3 and 8 years. CML in the blastic phase has the shortest survival rate time (less than 6 months) [31].

Apart from the phase of disease, a number of independent factors also serve as prognostic factors such as age, spleen 
size, liver size, and basophils count. Sokal et al in 1984 and Hashford et al in 1999 also identified some additional factors that affect survival rates in CML [32].

These 2 methods utilize mathematical models derived from similar clinical and laboratory parameters measured at diagnosis to identify 3 risk groups namely the low, intermediate, and high-risk group. The sokal scoring system was developed using patients treated with busulphan and hydroxyurea while taking into account the patient's age, blast count, spleen size, and platelet count at diagnosis [33]. The Hashford scoring system on the other hand was developed using patients treated with IFN- $\alpha$ while taking into consideration eosinophil, and basophil count in addition to values included in the sokal system [34]. Below are the mathematical expressions for both scores.

Sokal Score $=$ Exp. $[0.0116($ age -43.4$)+0.0345($ spleen size -7.51$)+0.188[($ platelet count $\div 700) 2-0.563]+$ 0.0887 (percentage blasts - 2.10)

Low risk $=<0.8$; Intermediate risk $=0.8-1.2$; High risk $=>1.2[33]$

Hasford/Euro Prognostic Score $=(0.6666 \times$ age $[0$ when age $<50$ years; 1 , otherwise $]+0.0420 \times$ spleen size $[\mathrm{cm}$ below costal margin] $+0.0584 \mathrm{x}$ blasts [\%] $+0.0413 \mathrm{x}$ eosinophils [\%] $+0.2039 \times$ basophils [0 when basophils $<3 \%$; 1 , otherwise] $+1.0956 \times$ platelet count [ 0 when platelets $<1500 \times 109 / \mathrm{L}$; 1 , otherwise]) x 1000.

Low Risk $=<780$; Intermediate Risk $=780-1480$; High Risk $=>1480$ [34]

The sokal scoring system also identified other significant prognostic indicators such as sex, eosinophil, and basophil count, serum lactate dehydrogenase activity, haematocrit and nucleated red blood cells in the peripheral blood. A new sokal score was devised by sokal and his colleagues in 1985 for young patients (lesser than 45 years) however this study was shown to be inadequate in discriminating between the intermediate and high-risk groups of survival by Hashford [35]

Hashford subsequently developed a new scoring system that showed better discrimination between risk groups with patients in the lowest risk group having a median survival time of 98 months, in the intermediate risk group it was 65 months and in the highest risk group, it was about 42 months [36]. This score has however not been used many times since its development due to it being replaced by the "Euro score" [37]. Euro score was the result of international project focused on the development of score valid specifically for CML patients treated with interferon. Another prognostic score was developed in an attempt to develop a score more suitable for patients treated with tyrosine kinase inhibitor called "EUTOS score" [38]. This was based on the end-point of achievement of complete cytogenetic response at 18 months of treatment with TKI. This end-point will most times predict progression-free survival of TKI-treated patients [39].

Some other scoring systems aside Hashford and Sokal have been developed such as "Tura score", "Cervantes score", Kantaranjian score" and "Grathwohl/EMBT score". Using these scoring systems, responses to therapy, survival, and risk of transformation, can be estimated. Grathwohl / EMBT score has been used to estimate the probability of a successful allogeneic Stem Cell Transplation (SCT) in individual patients with CML. This score includes a number of variables known to be indicative of a higher risk to die from SCT related complications. Most important 'riskindicators' in this regard appear to be age, type and sex of donor, and time from disease-onset. Pictorial representation of the scores and their factors is shown below [31].

Table 3. Overview of all prognostic scores historically used for assessment of CML patients [31].

\begin{tabular}{|c|c|c|}
\hline Score (reference) & Factors and their values; equation & Risk groups \\
\hline Tura & $\begin{array}{l}\text { splenomegaly }>15 \mathrm{~cm} \text { below costal margin, hepatomegaly }>6 \mathrm{~cm} \text { below costal margin } \\
\text { thrombocytopenia }<50 \times 109 / 1 \text { or thrombocytosis }>500 \times 109 / 1 \\
\text { leukocytosis }>100 \times 109 / 1 \text {, blasts in peripheral blood }>1 \% \\
\text { promyelocytes and myelocytes peripheral blood }>20 \%\end{array}$ & $\begin{array}{l}\text { Stage I (low risk): } \\
0 \text { - } 1 \text { factor } \\
\text { Stage II (intermediate risk): } \\
2 \text { - } 3 \text { factors } \\
\text { Stage III (high risk): } \\
4 \text { - } 6 \text { factors }\end{array}$ \\
\hline Cervantes & $\begin{array}{l}\text { splenomegaly, hepatomegaly, presence of erythroblasts in peripheral blood, myeloblasts in bone } \\
\text { marrow }>5 \%\end{array}$ & $\begin{array}{l}\text { Stage I (low risk): } \\
0 \text { - } 1 \text { factor } \\
\text { Stage II (intermediate risk): } \\
2 \text { factors } \\
\text { Stage III (high risk): } \\
3 \text { - } 4 \text { factors }\end{array}$ \\
\hline Kantarjian & $\begin{array}{l}\text { age } \geq 60 \\
\text { blasts in peripheral blood } \geq 3 \% \\
\text { blasts in bone marrow } \geq 5 \% \\
\text { basophils in peripheral blood } \geq 7 \% \\
\text { basophils in bone marrow } \geq 3 \% \\
\text { platelet count } \geq 700 \times 109 / 1 \\
\text { splenomegaly } \geq 10 \mathrm{~cm} \text { below costal margin } \\
\text { Accelerated phase: } \\
\text { blasts in peripheral blood } \geq 15 \% \\
\text { basophils in peripheral blood } \geq 20 \% \\
\text { blasts and promyelocytes in peripheral blood } \geq 30 \% \\
\text { platelet count } \leq 100 \times 109 / 1 \\
\text { cytogenetic clonal development }\end{array}$ & $\begin{array}{l}\text { Stage I: } \\
0 \text { - } 1 \text { factor } \\
\text { Stage II: } \\
2 \text { factors } \\
\text { Stage III: } \\
3 \text { or more factors } \\
\text { Stage IV: accelerated phase }\end{array}$ \\
\hline
\end{tabular}




\begin{tabular}{|c|c|c|}
\hline Score (reference) & Factors and their values; equation & Risk groups \\
\hline Sokal & $\begin{array}{l}\text { Exp }[0.0116(\text { age }-43,4)+0,0345 \text { (spleen size below costal margin }(\mathrm{cm})-7,51)+0,188 \text { ([platelet } \\
\text { count: } 700] 2-0,563)+0,0887 \text { (blasts in peripheral blood }(\%)-2,1)]\end{array}$ & $\begin{array}{l}\text { Low risk: }<0.8 \\
\text { Intermediate risk: } 0.8-1.2 \\
\text { High risk: }>1.2\end{array}$ \\
\hline Hasford & $\begin{array}{l}0,011 \times \text { age }+0,035 \times \text { spleen size below costal margin }(\mathrm{cm})+0,101 \times \text { erythroblasts in peripheral } \\
\text { blood }(\%)+0,11 \times \% \text { eosinophils in peripheral blood }(\%)+0,35 \times \text { gender }(\text { male }=2, \text { female }=1)\end{array}$ & $\begin{array}{l}\text { Low risk: }<0.8 \\
\text { Intermediate risk: } 0.8-1.2 \\
\text { High risk: }>1.2\end{array}$ \\
\hline Euro & $\begin{array}{l}(0,666 \times \text { age }[0 \text { if age }<50 \text { years otherwise } 1]+0,042 \times \text { spleen size below costal margin }(\mathrm{cm})+ \\
0,0584 \times \text { blasts in peripheral blood }(\%)+0,0413 \times \text { eosinophils in peripheral blood }(\%)+0,2039 \times \\
\text { basophils in peripheral blood }(\%)[0 \text { if basophils }<3 \% \text { otherwise } 1]+1,0956 \times \text { platelet count }[0 \text { if } \\
\text { platelet count }<1500 \times 109 / 1 \text { otherwise } 1]) \times 1000\end{array}$ & $\begin{array}{l}\text { Low risk: } \leq 780 \\
\text { Intermediate risk: }>780 \leq \\
1480 \\
\text { High risk: }>1480\end{array}$ \\
\hline EUTOS & $7 \mathrm{x}$ basophils in peripheral blood $(\%)+4 \mathrm{x}$ spleen size below costal margin $(\mathrm{cm})$ & $\begin{array}{l}\text { Low risk: } \leq 87 \\
\text { High risk: }>87\end{array}$ \\
\hline
\end{tabular}

\subsection{Treatment Options of CML}

The only curative treatment of CML today is stem cell transplantion with all other forms of therapy being noncurative although some of them may result in prolonged cytogenetic remission [40]. Monotherapy with a tyrosine kinase inhibitor (TKI) that targets the ABL1 kinase is currently regarded as standard treatment for the chronic and advanced phases of CML. Antimetabolites (such as cyarabine, hydroxyurea), alkylating agents, interferon alfa $2 \mathrm{~b}$, and steroids were earlier used for treating CML-CP, but these drugs have been replaced by imatinib. The blastic phase of CML may be treated either with a TKI alone or in combination with chemotherapy [40]. Prior to the introduction of tyrosine kinase inhibitors, the following treatment options were used against CML.

Busulphan is an oral alkylating agent generally used for conditioning before transplant procedures. Patients occasionally develop severe pancytopenia with bone marrow hypoplasia on standard dose. Other toxic effects include cutaneous pigmentation, pulmonary fibrosis and a wasting syndrome resembling hypo-adrenalism [41].

Hydroxyurea is a ribonucleotide reductase inhibitor that works by targeting relatively mature myeloid progenitors in a proliferative cycle. A few side effects of hydroxyurea at high dosage includes nausea, ulcer of the buccal cavity, skin rashes, diarrhea, and megaloblastic changes in the marrow [41].

Interferon-alpha (IFN- $\alpha$ ) is a member of a large family of glycoproteins with a much higher survival rate compare to hydroxyurea. Toxicity of IFN- $\alpha$ is common in older patients with side effects ranging from fevers, shivers, muscle aches etc. Patients might also develop intolerance to the drug on account of weight loss, lethargy, and anorexia [41].

With the adverse side effects related to the above stated treatment options, the approval of imatinib mesylate in December, 2002 by the US FDA has changed the natural history of CML changed dramatically.

\subsubsection{Imatinib}

Imatinib was the first tyrosine kinase inhibitor to be licensed by the United State FDA in 2001 for use in treatment of CML due to its ability to specifically target BCR-ABL caused by the Philadelphia chromosome translocation.
Imatinib is an oral 2 phenylaminopyrimidine derivative that acts as a tyrosine kinase inhibitor with selectivity for the ABL kinase. It is a potent competitive inhibitor of ATP binding to ABL kinase, as well as to the c-Kit and PDGF receptor tyrosine kinases. As a result, it specifically blocks the BCR-ABL fusion kinase, as well as activated c-Kit mutants in gastrointestinal stromal tumors (GIST), and preventing activation of mutations of the PDGF receptor tyrosine kinase in metastatic dermatofibrosarcoma protuberans. [42, 43]. Its effect on other cancers resulting from activation of ABL, c-kit, or PDGF (e.g., small cell lung cancer, prostate cancer, stromal and vascular tumors) is currently being evaluated. A few adverse effects of Imatinib are edema, nausea and vomiting, muscle cramps, musculoskeletal pain, diarrhea, and rash (occurring more frequently) with dry skin, alopecia, and abdominal distension occurring less frequently. Imatinib is known to be metabolized by cytochrome-P450, mostly the CYP3A4 and CYP3A5 isoforms, and to some extent CYP2D6, CYP2C9, CYP1A2 and CYP2C19. The active uptake of imatinib into the cells is mediated mainly by OCT1, OCTN2 transporters while its efflux is mediated

A study done by Hashford et al in 2004 revealed the estimated 10-year survival probability of imatinib to be $51 \%$ compared to $26 \%$ for IFN-a $[19,20]$ with another study carried out at Ile-Ife, Nigeria, by Durosinmi et al. showing a complete cytogenetic response (CCR) rate of 59\% and major cytogenetic response (MCR) of 35\%, achieved in six months in a median follow up of 25 months [44]. Another study also conducted in Italy in patients with CP CML who have failed IFN- $\alpha$ showed imatinib mesylate to effectively induce high CHR and cytogenetic response rates with relatively few side effects with CHR being 95\%, MCR 60\%, and complete cytogenetic remission 46\% [45]. A study by Adeagbo et al. showed ABCB1 C3435T had higher frequencies of complete haematological response (CHR), complete cytogenetic response (CCR) and major molecular response (MMR) [46]. A number of other factors contribute to the survival of patients such as patient's response to initial treatment, the time duration between the diagnosis and onset of Glivec therapy and also the use of conventional chemotherapy before the start of Glivec therapy

However, despite these promising results obtained in imatinib, several CML patients in Nigeria have developed resistance to the drug most probably due to increased 
genomic instability causing CML progenitor cells to acquire a number of genetic alterations. Identification of the molecular basis of resistance is important, because it could provide insight into disease progression and into the design of novel therapeutic strategies to prevent and overcome treatment resistance. CML patients with imatinib mesylate resistance can be divided into those with primary refractory disease, which frequently occurs in accelerated or blastic phase, and those who relapse after initial response, common in chronic phase patients. Due to the difficulty in measuring $\mathrm{BCR} / \mathrm{ABL}$ enzymatic activity in the blood, the level of phosphorylation of the $\mathrm{BCR} / \mathrm{ABL}$ substrate protein called CRKL, is used to determine the endpoint of tyrosine kinase activity. Patients with high levels of CRKL phosphorylation despite treatment with imatinib usually show resistance based on 3 different mechanisms namely: BCR/ABL gene amplification, $\mathrm{BCR} / \mathrm{ABL}$ mutations, and high plasma levels of alphalacid glycoprotein (AGP). In $\mathrm{BCR} / \mathrm{ABL}$ gene amplification, an increase in $\mathrm{BCR} / \mathrm{ABL}$ expression caused by double $\mathrm{Ph}$ ' chromosome for this resistance. Consequently, $\mathrm{BCR} / \mathrm{ABL}$ mutation arises from mutation in the amino acid of ATP binding sites of the tyrosine kinase domain preventing imatinib from binding to BCR/ABL thereby causing lack of inhibition to tyrosine kinase activity. The $3^{\text {rd }}$ mechanism of resistance involves high plasma levels of AGP which has been shown to bind imatinib at physiological concentrations thereby blocking its ability to inhibit BCR/ABL kinase activity in a dose dependent manner [47]. In the long-term, only $70 \%$ of CML patients taking imatinib maintain a perfect response. Development of resistance to imatinib over the years has led to the development of new tyrosine kinase inhibitor drugs with higher survival rate for CML patients. Examples of these drugs are: Nilotinib, Dasatinib, Bosutinib, and Ponatinib.

\subsubsection{Nilotinib}

This is a second-generation TKI effective against imatinib resistant patients. In addition to $\mathrm{BCR}-\mathrm{ABL} 1$, it also inhibits PDGFR and c-kit. It is active against a range of imatinibresistant kinase domain mutations, except for T315I, F359V/C, E255K/V and Y253H/F. Common associated biochemical abnormalities include elevations in serum liver enzymes, lipase and amylase, and hyperbilirubinaemia with pancreatitis occurring less frequently. Incidence of cross intolerance with imatinib is low. Although all TKIs can increase survival rate of patients, the effect of nilotinib is perhaps the best documented. Nilotinib is currently indicated for second-line treatment of CML-CP and CML-AP in patients who have resistance or intolerance to at least one TKI at a dose of $400 \mathrm{mg}$ BID. There is currently inadequate clinical evidence to recommend its use in Ph+ ALL or CMLBP. The data surrounding its frontline use came from the ENESTnd study, which showed CML-CP patients treated with frontline nilotinib (at either $300 \mathrm{mg}$ BID or $400 \mathrm{mg}$ BID) have decreased risk of disease transformation to AP/BP, reduced risk of kinase domain mutation acquisition, and higher achievement of MMR/MR when compared to imatinib treated patients Nilotinib-treated patients have higher incidence of hyperglycaemia and dyslipidaemia, and accelerated development of arterial occlusive disease is an emerging concern. This manifests as ischaemic heart disease, strokes and peripheral arterial occlusive disease, and appears to be more common in both nilotinib arms compared to the imatinib arm in the ENESTnd study [48, 49].

\subsubsection{Dasatinib}

Dasatinib is a second-generation TKI and a dual SRC/ABL inhibitor, though it also inhibits a number of other tyrosine kinases. It is approved for frontline treatment of CML-CP, as well as cases resistant or intolerant to at least one prior TKI in all phases, and for second-line use in Philadelphia positive ALL. It has the greatest in vitro activity against BCR-ABL1 amongst the three TKIs approved for frontline treatment and, unlike imatinib, dasatinib can bind to BCR-ABL1 in active conformation. The dosage for Dasatinib is $100 \mathrm{mg}$ for $\mathrm{CP}$ and $140 \mathrm{mg}$ for advanced phases taken with or without food. In the Phase III DASISION study, dasatinib-treated patients had superior achievement of MMR (34\%) and CR (77\%) in 24 months [48, 49] compared to imatinib treatment, though no significant difference in overall survival and progression-free survival was demonstrated. Interestingly, the number of patients who developed kinase domain mutations was similar in both arms, with a higher number of T315I cases in dasatinib treated patients.

\subsubsection{Bosutinib}

Bosutinib is a second generation TKI indicated for the treatment of CML patients with intolerance or resistance to at least one other TKI, for all disease phases. It is administered as a single daily dose of $500 \mathrm{mg}$ daily. In the second-line setting, approximately 50\% of CML-CP patients who switch from imatinib to bosutinib will achieve CCyR. This fall to less than one-third for patients who have failed two previous TKIs, and is $25 \%$ and $20 \%$ in accelerated and blastic phases, respectively. Clinical evidence suggests inferior responses for patients with the V299L, L248V, F359C (and perhaps G250E) mutations. The T315I mutant clone is totally resistant to bosutinib. Evaluation of bosutinib's efficacy in the frontline setting is ongoing. Gastrointestinal side effects, including diarrhoea, nausea, abdominal pain and vomiting are common and experienced by up to $80 \%$ of patients. Other common non-haematological toxicities include rash, headache and liver enzyme elevations [50].

\subsubsection{Ponatinib}

Ponatinib is a third-generation TKI, and is the only drug with activity against the T315I mutation. Cases with compound mutations (where more than one kinase domain point mutation is present in the same BCR-ABL1 transcript), especially those involving the $\mathrm{E} 255 \mathrm{~K} / \mathrm{V}$ mutation, are potentially resistant to this drug. Ponatinib is effective in patients with resistance or intolerance to at least one previous line of treatment in all phases of CML disease, as well as $\mathrm{Ph}+-\mathrm{ALL}$, especially in patients with T315I as the only known mechanism of resistance. Rash, elevated lipase, 
pancreatitis and headaches seem to be common nonhaematological toxicities of the drug. Of significant concern, $27 \%$ of patients who participated in early clinical studies reported having had either arterial or venous thrombotic events. In light of emerging toxicities, a Phase III study comparing imatinib to ponatinib as frontline therapy was prematurely terminated, though ponatinib retains marketing approval in the EU and US for $\mathrm{Ph}+$ leukaemias with the T315I mutation, and in cases where other TKIs have no real prospect of therapeutic success. This drug should be used with extreme caution and at the lowest effective dose in patients with risk factors for vascular disease, and patients with a history of vascular events may not be suitable candidates for this drug [51].

The only "curative" treatment of CML in the world today is stem cell transplantation.

\section{Mutations to Tyrosine Kinase Inhibitors in CML}

Mutations in the $\mathrm{BCR} / \mathrm{ABL}$ kinase domain may cause resistance to tyrosine kinase inhibitors in CML patients. Since the first imatinib-resistant cases, point mutations in the kinase domain $(\mathrm{KD})$ of $\mathrm{BCR} / \mathrm{ABL}$ have been identified. A study conducted by Willis et al showed T3151 as the most frequent mutation among imatinib treated patients, followed by $\mathrm{Y} 253 \mathrm{H}, \mathrm{Q} 252 \mathrm{H}$, and F359V being less frequent. Nilotinib is active against a range of imatinib-resistant kinase domain mutations, except for T315I, F359V/C, E255K/V and $\mathrm{Y} 253 \mathrm{H} / \mathrm{F}$. Dasatinib and nilotinib when used as a second line treatment of imatinib-intolerant or -resistant patients, leads to the emergence of mutations [T315I, V299L, T315A, F317L/V/I/C] for Dasatinib or nilotinib [E255K/V, Y253H, $\mathrm{F} 359 \mathrm{~V} / \mathrm{C} / \mathrm{I}]$. However, their use as first line drugs yields fewer mutations in $\mathrm{T} 315 \mathrm{I}$ and $\mathrm{E} 255 \mathrm{~K}$ respectively [52]. Clinical evidence has shown Bosutinib to have inferior responses for patients with the V299L, L248V, F359C and G250E mutations while being totally resistant to T3151 mutation. Ponatinib is a third-generation TKI, and is the only drug with activity against the T315I mutation. Therefore, In case of a T315I mutation, which is highly resistant to imatinib, dasatinib, and nilotinib, there are no pharmacologic opportunities other than investigational compounds in phase $1 / 2$ clinical development. - In case of V299L, T315A, or $\mathrm{F} 317 \mathrm{~L} / \mathrm{V} / \mathrm{I} / \mathrm{C}$ mutations, nilotinib is probably more effective than dasatinib. - In case of $\mathrm{Y} 253 \mathrm{H}, \mathrm{E} 255 \mathrm{~K} / \mathrm{V}$, or F359V/C/I mutations, dasatinib is probably more effective than nilotinib. - In case of any other mutation, dasatinib and nilotinib are likely to be similarly effective [53].

HAEMOTOPOIETIC STEM CELL TRANSPLANTATION (HSCT): this involves the intravenous infusion of autologous allogenic stem cells to reestablish hematopoietic function in patients whose bone marrow or immune system is compromised [54]. HSCT can be classified according to the relationship between the patient and the donor and by the anatomic source of the stem cells.
Patient-donor relationship could be syngeneic, autologous or allogeneic. Based on sources of stem cells, we have bone marrow stem cells and peripheral blood stem cells. It is often done or reserved for patients with advanced phases of the disease or therapeutic failure to all available TKI's. Allogenic hematopoietic stem cell transplantation outcomes may be predicted using the EMBT risk score. Patients in tropical regions like Nigeria undergo significant parasitological workup/ prophylaxis before starting HSCT. Allogeneic SCT is advised for all $\mathrm{Ph}+$ diseases and other high risk ALL patients. Most experts agree that young patients with high risk should have HSCT done. The disease free survival rate at 5 years are $50 \%, 30-40 \%$, and $15-20 \%$ for patients treated at chronic, accelerated, and blast phases respectively. Problems associated with HSCT in other diseases also apply to CML. Transplant related mortality, graft vs host disease and relapse-related mortality are significant problems. Use of TKI's after HSCT is also encouraged. Detection of residual CML in accelerated and blastic phase of the disease is done through marrow real time quantitative PCR (RQ-PCR) [55].

\section{Blood Transfusion for Leukemia Patients}

In addition to cancer treatments such as chemotherapy and stem cell transplants, leukemia patients may receive supportive treatments to help them cope with the illness. Since both the illness and chemotherapy can disrupt red blood cell development, causing anemia, thrombocytopenia, or neutropenia, people with leukemia often receive whole blood or blood products to supplement their treatment. Similarly, patients undergoing stem cell transplantation are given heavy doses of chemotherapy, which depletes natural red blood cell reserves. This can be counteracted with transfusing the patients with red blood cells [56]. Some typical examples of blood products transfused to leukemia patients are: red cell transfusion, platelet transfusion, granulocyte transfusion, plasma transfusion, albumin transfusion and intravenous gamma globulin. Red cell transfusion is given to anaemic patients to prevent shortness of breath and rapid heartbeat. Platelet transfusion is given to prevent excessive bleeding. Granulocytes are transfused to help patients with low white blood cells fight off antibiotic resistant infections. Fresh frozen plasma and cryoprecipitate may be transfused to patients with low level of blood clotting factors. Albumin is mainly given to patients with severe liver malfunction. Gamma globulins are usually given to patients undergoing stem cell transplant to reduce the risk of cytomegalovirus infection [57].

\section{Prospects and Challenges}

Although, there has been significant improvement in the treatment outcome of CML patients in the last decade, several improvements can still be made. In many developing countries such as Nigeria, antimetabolites (hydroxyurea) are 
still being used in many centres for the initial cytoreduction of CML to a complete haematological response. This is due the fact that only one Imatinib centre is available in the country at no cost to patient's courtesy of Novartis, Max foundation and Axios International. Propolis lecithin has shown to be promising and is currently under research.

Nigeria being the most populous black nation with a population of over 200 million people is exceptionally placed to be a corner stone for HSCT in the Africa in the coming years [54]. However, to achieve this, some actions have to be taken one of which includes development of more HSCT centres in the country with only a few in private health facilities being currently functional. This alongside other challenges such as limited expertise, erratic power supply, poor infrastructure, maintenance capabilities, and poor political support have limited its advancement in Nigeria. There is therefore a need to invest more resources to train more skilled personnel, acquire more equipment and maintain them better. It is equally important to improve on health insurance scheme, and sensitize policy makers on the need for these services and improved healthcare funding.

\section{Conclusion and Recommendation}

Conclusively, chronic myeloid leukemia is a curable disease with a high survival rate if detected early, but becomes increasingly aggressive and resistant to therapy as it progresses to accelerated and blastic phase. This has led to improvements in methods used for accurate diagnosis of the disease.

However, developing countries that have low resource economy like Nigeria do not have enough technical expertise and equipment to facilitate this. Treatment options should be delivered on a person to person basis based on their clinical symptoms displayed by the patient. However, hematopoietic stem cell transplantation remains the only curative treatment approach, and should be considered as long as the patient can afford it.

Development of resistance to TKI's over the years has led experts to look for additional targeted and conventional antileukemic drugs.

We therefore recommend that the Federal Government of Nigeria provide more support in the form of funding, legislative policies, opening more centres for Imatinib treatment of CML in the country. Also, improving capacity building and power generation will help health facilities performing these procedures, thereby improving the survival rate of CML patients and reduce medical tourism to other developed countries.

\section{References}

[1] Durosinmi M. A. Haemato-Oncology Chemotherapy. pp 1517.

[2] J. V. Melo, T. P. Hughes, and J. F. Apperley, "Chronic myeloid leukemia, The American Society of Hematology, pp. 132-152,
2003. Faderl S, Kantarjian HM, Talpaz M. Chronic myelogenous leukemia: update on biology and treatment. Oncology. 1999; 13: 169-184.

[3] Oyekunle A. A., Durosinmi, M. A., Bolarinwa R. A. and Owojuyigbe T. Chronic Myeloid Leukemia in Nigerian Patients: Anemia is an Independent Predictor of Overall Survival. Clinical Medicine Insights: Blood Disorders 2016: 9 9.

[4] Lateef Salawu and Norah O. Akinola Lihteh Wu, Joaquin Martinez, Andrew W. Opthalmologic Manifestations of Leukemias. Medscape. 2020.

[5] Caroline E. O and Olatunji E. Trends in Pattern of Leukaemia incidence in a Tertiary Hospital in Nigeria: 1990-2004. Journal of Medicine and Biomedicine research 2006. (5): 4449.

[6] Boma PO, Durosinmi MA, Adediran 1A, Akinola NO, Salawu I. Clinical and prognostic features of Nigerians with Chronic Myeloid Leukemia. Niger PostgradMedJ. 2006; 13: 47-52.

[7] Oyekunle AA, Zander AR, Binder M, et al. Outcome of allogeneic SCT in patients with chronic myeloid leukemia in the era of tyrosine kinase inhibitor therapy. Ann Hematol. 2012; 92 (4): 487-496.

[8] Michael W. D. Chronic Myeloid Leukemia: An Historical Perspective. Haematol Educ Prog. 2008: (1): 418.

[9] Nwagu M. U., Awodu O. A. Chronic Myelogenous Leukemia - A Review of Pathophysiology, Clinico-Laboratory Features and Emerging Treatment Options. Nig Hosp Pract. (6), 2010, 93-96.

[10] Victor Hoffbrand, Douglas Higgs, David Keeling, Atul B Mehta Postgraduate Haematology. pp 419-437.

[11] Maren Rohrbacher and Joerg Hasford. Etiology and Epidemiology of Chronic Myeloid Leukemia. Neoplastic Diseases of the Blood, 2013 (2): 11-17.

[12] Goldman J. M., Melo J. V. Chronic myeloid leukemia Advances in biology and new approaches to treatment. $N$ Eng J Med. 2003; 349: 1451-1464.

[13] Talpaz M., Shah N. P., Kantarijan (2006). Dasatinib in imatinib-resistant Philadephia chromosome-positive leukemias. N. Engl. J. Med. 354, 2531-41.

[14] Ishikura H, Yufu Y, Yamashita S, Abe Y, Okamura T, Motomura S, Nishimura J, Nawata H (1997). Biphenotypic blast crisis of chronic myelogenous leukemia: abnormalities of p53 and retinoblastoma genes. Leuk Lymphoma 25: 573-578.

[15] Goldman J. M. Monitoring minimal residual disease in BCRABL positive chronic myeloid leukemia in imatinib era. Current Opinion in Haematology.

[16] Bain B. (2003). Cytogenetic and molecular genetic aspects if eosinophilic leuekemias. British Journal of Haematology. 122: $173-9$.

[17] Skorski T (2002) BCR/ABL regulates response to DNA damage: the role in resistance to genotoxic treatment and in genomic instability. Oncogene 21: 8591-8604.

[18] Janowska-Wieczorek A, Majka M, Marquez-Curtis L, Wertheim JA, Turner AR, Ratajczak MZ (2002) Bcr-abl positive cells secrete angiogenic factors including matrix metalloproteinases and stimulate angiogenesis in vivo in Matrigel implants. Leukemia 16: 1160-1166. 
[19] Sillaber C., Mayerhofer M., Agis H., Sagaster V., Mannhalter V., Sperr W. R., Geissler K., and Valent P. Chronic myeloid leukemia: Pathophysiology, diagnostic parameters, and current treatment concepts. Wien Klin Wochenschr (2003) 115/13-14: 485-504.

[20] Vardiman JW, Pierre R, Thiele J, Imbert M, Brunning RD, Flandrin G (2001) Chronic myelogenous leukaemia. In: Jaffe ES, Harris NL, Stein H, Vardiman JW (eds) Tumours of haematopoietic and lympoid tissues. IARC Press, Lyon, pp 20-26.

[21] Tefferi A, Thiele J, Orazi A et al. Proposals and rationale for revision of the world health organization diagnostic criteria for polycythemia vera, essential thrombocythemia and primary myelofibrosis: recommendation from an ad hoc international expert panel. Blood 2007; 110: 1092-1097.

[22] Tefferi A. Classification, diagnosis and management of myeloproliferative disorders in the JAK2V617Fera. Hematology Am Soc Hematol Educ Program 2006; 240-245.

[23] Shirish M. K. Essentials of Haematology. $2^{\text {nd }}$ edition 2013.pp 284-287.

[24] Baccarani M, Deininger MW, Rosti G et al. (2013) European Leukemia Net recommendations for the management of chronic myeloid leukemia: 2013. Blood 122: 872-84.

[25] Kyrsiak K., Christopher M., Skidmore Z., Demeter R., Magrini V., Kunisaki J., Laughlin M., Duncavage E., Miller C., Ozenberger B., Griffith M., Wartman L. A genomic analysis of Philadelphia chromosome-negative AML arising in patients with CML (2016). Blood Cancer Journal. 6; 413.

[26] Solary E., Itzykson R. How I treat chronic myelomonocytic leukemia. Blood. (2017). 130 (2): 126-136.

[27] Szuber N., Tefferi A. Chronic neutrophilic leukemia: new science and new diagnostic criteria (2018). Blood Cancer Journal. 8: 19.

[28] Thakral Beenu, Wang A. Haematology and Coagulation. A comprehensive review for board preparation, certification, and clinical practice. 2015, pages 93-115.

[29] Andolina J. R., Neudorf S. M., Corey S. J. (2012) How I treat childhood CML. Blood 119: 1821-30.

[30] Apperley J (2009) CML in pregnancy and childhood. Best Practice in Research and Clinical Haematology 22: 455-74.

[31] Faber E. (2015) Prognostic Scoring Systems in CML Improvement Impossible? Journal of Leukemia, S1: 001.

[32] Verstovsek S, Kantarjian H, Manshouri T, Cortes J, Giles FJ, Rogers A, Albitar M (2002) Prognostic significance of cellular vascular endothelial growth factor expression in chronic phase chronic myeloid leukemia. Blood 99: 2265-2267.

[33] Sokal JE, Cox EB, Baccarani M, Tura S, Gomez GA, et al. (1984) Prognostic discrimination in "good-risk" chronic granulocytic leukemia. Blood 63: 789-799.

[34] Hasford J, Pfirrmann M, Hehlmann R, Baccarani M, Guilhot F, Mahon FX, Kluin-Nelemans HC, Ohnishi K, ThaleeJ, Steegmann JL (2003) Prognosis and prognostic factors for patients with chronic myeloid leukemia: nontransplant therapy. Semin Hematol 40: 4-12.

[35] Hasford J, Ansari H, Pfirrmann M, Hehlmann R (1996) Analysis and validation of prognostic factors for CML. German CML Study Group. Bone Marrow Transplant 17 Suppl 3: S49-54.
[36] Hasford J, Baccarani M, Hoffmann V, Guilhot J, Saussele S, et al (2011) Predicting complete cytogenetic response and subsequent progression-free survival in 2060 patients with CML on imatinib treatment: the EUTOS score. Blood 118: 686-692.

[37] Marin D, Ibrahim AR, Goldman JM (2011) European Treatment and Outcome Study (EUTOS) score for chronic myeloid leukemia still requires more confirmation. J Clin Oncol 29: 3944-3945.

[38] Jabbour E, Cortes J, Nazha A, O'Brien S, Quintas-Cardama A, et al. (2012). EUTOS score is not predictive for survival and outcome in patients with early chronic phase chronic myeloid leukemia treated with tyrosine kinase inhibitors: a single institution experience. Blood 119: 4524-4526.

[39] Hoffmann VS, Baccarani M, Lindoerfer D, Castagnetti F, Turkina A, et al. (2013) The EUTOS prognostic score: review and validation in 1288 patients with CML treated frontline with imatinib. Leukemia 27: 2016-2022.

[40] Druker BJ, Guilhot F, O'Brien SG et al. Five-year follow-up of patients receiving Imatinib for Chronic Myeloid Leukaemia. New England Journal of Medicine. 2006; 355 (20): 2408 2417.

[41] Goldman J. M., Druker B. Chromic myeloid leukemia: current treatment options. Blood. 98: 2039-42.

[42] Hasford J, Pfirrmann M and Hocchaus A. How long will chronic myeloid leukemia patients treated with imatinib mesylate live? Leukemia 2005; 19: 497-499.

[43] Christian S., Matthias M., Hermine A., Verena S., Christine M., Wolfgang R., Klaus G., and Peter V. Chronic myeloid leukemia: Pathophysiology, diagnostic parameters, and current treatment concepts. (2003) 115/13-14: 485-504.

[44] Deininger, M. W. N., Druker, B. J., 2003. Specific targeted therapy of chronic myelogenous leukemia with imatinib. Pharmacological Reviews, 55 (3), 401-423.

[45] Rubin, B. P., Schuetze, S. M., Eary, J. F., Norwood, T. H., Mirza, S., Conrad, E. U., Bruckner, J. D., 2002. Molecular targeting of platelet-derived growth factor B by imatinib mesylate in a patient with metastatic dermatofibrosarcoma protuberans. J. Clin. Oncology, 20 (17), 3586-3591.

[46] Durosinmi et al. The use of imatinib mesylate in Nigerians with chronic myelogenous leukemia. Cellular Therapy and Transplantation (CTT) 2008; vol 1 No. 2.

[47] Guido Marcucci, Danilo Perrotti, and Michael A. Caligiuri. Understanding the Molecular Basis of Imatinib Mesylate Therapy in Chronic Myelogenous Leukemia and the Related Mechanisms of Resistance. 9, 1248-1252, April 2003.

[48] Adeagbo B. A, Bolaji O. O., Olugbade T. O, Durosinmi M. A., Bolarinwa R. A., Masimirembwa C.

[49] Gambacorti-Passerini, C. B., Gunby, R. H., Piazza, R., Galietta, A., Rostagno, R., and Scapozza, L. Molecular mechanisms of resistance to imatinib in Philadelphiachromosome-positive leukaemias. Lancet Oncol., 4: 75-85, 2003.

[50] Branford S, Melo JV and Hughes TP (2009) Selecting optimal second-line tyrosine kinase inhibitor therapy for chronic myeloid leukemia patients after imatinib failure: does the BCR-ABL mutation status really matter? Blood 114: 5426-35. 
[51] Gotlib J, Maxson JE, George TI and Tyner JW (2013) The new genetics of chronic neutrophilic leukemia and atypical CML: implications for diagnosis and treatment. Blood 122: 1707-11.

[52] Hagop M. Kantarjian et al. Long-term outcomes with frontline nilotinib versus imatinib in newly diagnosed chronic myeloid leukemia in chronic phase: ENESTnd 10-year analysis. Leukemia (2021) 35: 440-453.

[53] Simona S., Andreas H., Franck E., Franz G., Thoralf L., Giuseppe S., Fabrizio P., Martin C., Thomas E., Rosti G., Porkka K., Baccarani M., Nicholas C. P. and Giovanni Martinelli. BCR-ABL kinase domain mutation analysis in chronic myeloid leukemia patients treated with tyrosine kinase inhibitors: recommendations from an expert panel on behalf of European LeukemiaNet. Blood, 2011 volume 118, number 5.
[54] Oyekunle A. A. Haematopoietic Stem Cell transplantation: Prospects and Challenges in Nigeria. Annals of Ibadan Post graduate medicine. Vol. 4. No 1 June 2006.

[55] Oyekunle A. A. Recent Advances in Stem Cell Biology: Implications for Tropical Haematology Practice. Annals of Tropical Pathology Vol. 2 No 1 June, 2011.

[56] Körbling M, Freireich EJ. Twenty-five years of peripheral blood stem cell transplantation. Blood 2011; 117: 6411-6.

[57] Vamvakas EC, Blajchman MA. Transfusion-related mortality: the ongoing risks of allogeneic blood transfusion and the available strategies for their prevention. Blood 2009; 113: 3406-17. 